\title{
Adaptation of microscopy with ultraviolet surface excitation for enhancing STEM and undergraduate education
}

Chi Z. R. Huang

Ronald W. Wood

Stavros G. Demos 


\title{
Adaptation of microscopy with ultraviolet surface excitation for enhancing STEM and undergraduate education
}

\author{
Chi Z. R. Huang, ${ }^{a, b}$ Ronald W. Wood, ${ }^{c}$ and Stavros G. Demos ${ }^{b, *}$ \\ aUniversity of Rochester, Department of Biomedical Engineering, Rochester, New York, United States \\ bUniversity of Rochester, Laboratory for Laser Energetics, Rochester, New York, United States \\ 'University of Rochester, School of Medicine and Dentistry, Departments of Obstetrics and Gynecology, Urology, \\ and Neuroscience, Rochester, New York, United States
}

\begin{abstract}
Microscopy with ultraviolet surface excitation (MUSE) is investigated as a means to enhance curricula and education in the life sciences based on simplicity of use, the incorporation of inexpensive hardware, and the simplest methods of tissue preparation. Ultraviolet excitation in effect replaces tissue sectioning because it penetrates only a few micrometers below the tissue surface at the single cell level, preventing the generation of outof-focus light. Although tissue autofluorescence may be used, image quality and content can be enhanced by a brief immersion in a solution of nontoxic fluorescent dyes that selectively highlight different cellular compartments. Safe mixed-dye powder combinations have been developed to provide students who have minimal lab proficiencies with a one-step tissue staining process for rapid tissue preparation. $\odot$ The Authors. Published by SPIE under a Creative Commons Attribution 3.0 Unported License. Distribution or reproduction of this work in whole or in part requires full attribution of the original publication, including its DOI. [DOI: 10.1117/1.JBO.23.12.121603]
\end{abstract}

Keywords: imaging systems; fluorescence microscopy; medical and biological imaging; tissue characterization.

Paper 180227SSR received Apr. 17, 2018; accepted for publication Jun. 8, 2018; published online Jul. $27,2018$.

\section{Introduction}

Wide-field light microscopy is an iconic tool for introducing students to the microstructure of living organisms in biology education. Because of their versatility and low price, widefield light microscopes have been widely adopted by schools worldwide. ${ }^{1}$ Tissue light scattering makes direct imaging of a cellular structure impossible because the out-of-focus signal is much stronger and image contrast between cellular compartments is very limited. Consequently, viewing biological samples is limited to prepared slides or to samples from onion or cheek scraping. ${ }^{2}$ To examine cell membrane and nuclei, even onion skin must be cut to achieve a single layer of cells; this is a nontrivial task that cannot be accomplished in a brief classroom exercise. Therefore, students often find that exploring cellular anatomy is a frustrating experience. Urban community college students often leave such courses, subsequently setting them up for lower levels of achievement. ${ }^{3}$

Successful experiences are necessary to sustain educational progress, ${ }^{4}$ and anatomy remains a vital part of life-science education. ${ }^{5}$ In dissection, classroom students often study only major organ structures and typically dispose of specimens without examining their structure at a cell level. ${ }^{1}$ Prepared microscope slides and textbook images can be inadequate in stimulating interest in the life sciences or in establishing the self-confidence necessary to motivate pursuing a career in related fields. Point-by-point raster scanning imaging methods can be intensely rewarding by offering optical sectioning and

*Address all correspondence to: Stavros G. Demos, E-mail: sdemos@lle rochester.edu rapid imaging of tissue microstructure, ${ }^{6-12}$ but they are too complex and expensive for routine classroom use. Conventional histological staining, e.g., hematoxylin and eosin (H\&E), relies on fixation, embedding, sectioning, slide mounting, and processing by multiple immersions; sharp instruments, hot materials, and toxic solvents are routine hazards in histology labs_risks that must be minimized to meet classroom safety requirements. Ideally, students should be able to perform the entire process safely and quickly and must utilize tissue specimens commonly used in high school and undergraduate education, either freshly euthanized or obtained from biological supply houses.

Microscopy with ultraviolet surface excitation (MUSE) $)^{13}$ provides near-surface images of tissues without the need for laborious and prolonged tissue processing. Specimens may be quickly dyed and rinsed before placement in a wide-field epifluorescence microscope with the tissue surface flattened under slight pressure with a thin transparent window. An ultraviolet (UV) (such as an $270 \mathrm{~nm}$ ) light-emitting diode (LED) illuminates the tissue at an oblique angle, avoiding propagation through the microscope's optical elements, and fluorescent dyes emit visible fluorescence typical of each dye. Therefore, one UV excitation wavelength can excite two or more dyes concentrating in different cellular compartments, typically cytoplasm and nuclei. Because tissue penetration at UV wavelengths is very shallow, ${ }^{14}$ the signal is localized within the depth of a single cell. Resultant images can give extensive surface detail reminiscent of scanning electron microscopy that also resembles conventional histopathology. ${ }^{13}$ In effect, images can be obtained in a few minutes that would otherwise take at least several hours to achieve with conventional tissue processing. 
The current work reports our efforts to investigate MUSE imaging procedures that could be easily and inexpensively adopted for STEM (science, technology, engineering, and mathematics) and undergraduate science education. In contrast to the application of MUSE in the medical field, ${ }^{13}$ MUSE imaging for education can afford lower spatial resolution, which translates into even simpler and less-expensive optical designs. Within this context, we investigate MUSE microscopy using only tissue autofluorescence, and we discuss the benefits of tissue staining using widely available and safe stains. We also discuss our approach to optimize the tissue staining process by developing a stain mixture in powder form contained within a gelatin capsule to facilitate rapid one-step staining. We also demonstrate approaches to digital imaging processing and formats of image acquisition that improve image quality and enhance STEM education.

\section{Materials and Methods}

The epifluorescence microscope shown in Fig. 1 was assembled from readily available parts. A variety of microscope objectives were explored including $5 \times$ and $10 \times$ long-working-distance objectives and $4 \times$ and $10 \times$ international standard microscope objectives (Edmund Optics). Also, various chargecoupled-device (CCD) and complementary metal-oxide semiconductor (CMOS) cameras having pixel sizes between 2.4 and $4.6 \mu \mathrm{m}$ (different manufacturers) were tested, whereas the excitation light was provided by a $600-\mu \mathrm{W}, 270-\mathrm{nm}$ UV LED (QPhotonics). The LED source was typically focused on an $\sim 4-\mathrm{mm}^{2}$ area using a ball lens, resulting in a power density of $\sim 10 \mathrm{~mW} / \mathrm{cm}^{2}$. The UV excitation was delivered at an oblique angle to the sample's surface through the sapphire window. In this arrangement, the camera captures an image of a $\sim 1-\mathrm{mm}^{2}$ section with an optical resolution of $\sim 1 \mu \mathrm{m}$ and a digital registration of $\sim 2$ pixels $/ \mu \mathrm{m}$ or higher (depending on the camera/objective combinations).

Tissue samples were cut to $\sim 5$-mm-thick blocks using a surgical dissecting knife. For autofluorescence imaging, the tissues blocks were rinsed and loaded onto a linear stage without any additional preparation. The entire sample holder (including the optical window holder) was mounted on a three-axis translation

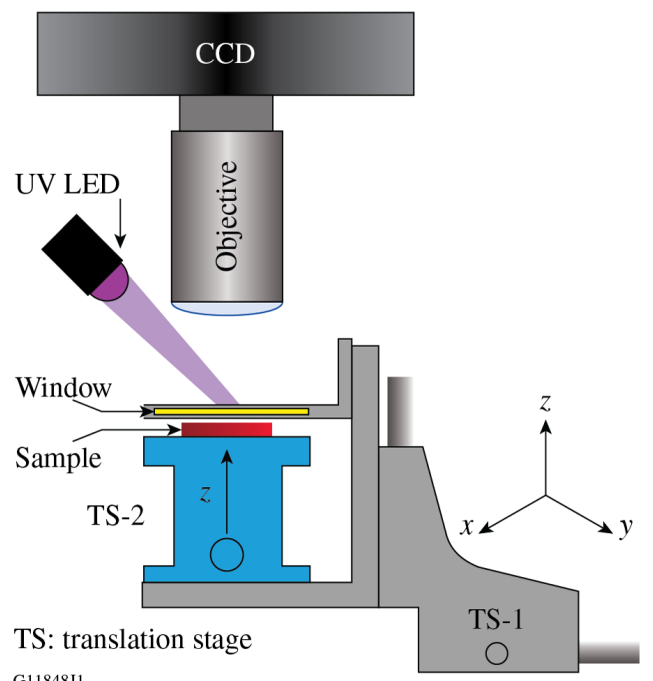

Fig. 1 Schematic layout of the MUSE microscope experimental configuration. Various types of microscope objectives and CCD/CMOS cameras were used. TS, translation stage. stage (TS-1) for focusing and horizontal movement. The sample was raised using translation stage TS-2 until the tissue surface was in contact with a sapphire optical window that created a flat surface at the microscope's image plane. A variety of tissues that might be used in STEM contexts were imaged, including fresh frog (ranine) organs from tissue obtained in the local market and preserved fetal pig (porcine) obtained from a biological supply house that supplies educational materials to schools and colleges. Comparable images can be obtained from laboratory animals (results not shown). Furthermore, onion tissue was imaged for direct comparison with the classical experiments of imaging onion cells.

Experiments using stained tissue were performed using fresh or formalin-fixed tissues that were briefly exposed to fluorescent dyes that localize in different cellular compartments and emit visible light. Several nontoxic fluorescent dyes were evaluated over a range of concentrations in deionized water, including DAPI, Hoechst 33342, and propidium iodide for nuclear staining and eosin $\mathrm{Y}$ and fluorescein as cytoplasmic counterstains. After each exposure, the samples were rinsed in a dye solution to remove excess dye. The emission spectral profiles of each dye, when simultaneously applied to a tissue, was selected to provide maximum compartmental separation. Samples were submerged in stain solutions for varying durations to optimize image quality; specific concentrations and immersion durations are reported in context below.

\section{Results and Discussion}

\subsection{Autofluorescence Imaging}

The LED excitation was sufficient to generate autofluorescence microscopic images of rinsed fresh tissues using exposure times of a few seconds (typically between 1 and $4 \mathrm{~s}$, depending on objective and camera selection). Figure 2 shows representative images from the surface of tissue sections having a thickness of the order of $5 \mathrm{~mm}$. Specifically, Fig. 2(a) shows the image of a thin section of onion tissue capturing the structure of the onion cells and their nuclei. In contrast to the laborious effort to image a thin section of onion under white-light microscopy, imaging with MUSE was very simple, involving a clean cut of a $5-\mathrm{mm}$ section of the onion tissue placed under the MUSE microscope. The images of the onion cells were readily accessible, showing the nuclei and membranes as features of higher intensity.

The image shown in Fig. 2(b) captures the structure of ranine kidney tubules as observed through the kidney membrane. The inset image of a single tubule at higher magnification shows that cell nuclei appear as lower-intensity features; similar images have been generated using laser excitation sources. ${ }^{15}$ The demonstration of such imaging using simple, inexpensive instrumentation is critical for the translation of this technology in the educational setting. The images of cardiac [Fig. 2(c)] and liver tissues [Fig. 2(d)] demonstrate that autofluorescence MUSE imaging can reveal the microstructure of various organs; note in Fig. 2(c) that the $\sim 10-\mu$ m-diam dark features are blood cells.

\subsection{Fluorescent Stain Evaluation}

Stains enhance the visualization of the tissue microstructure. A typical example is demonstrated in Fig. 3 showing (a) an autofluorescence image of preserved pig lung and (b) an image of a similar location following immersion in $500 \mu \mathrm{g} / \mathrm{mL}$ of Hoechst 

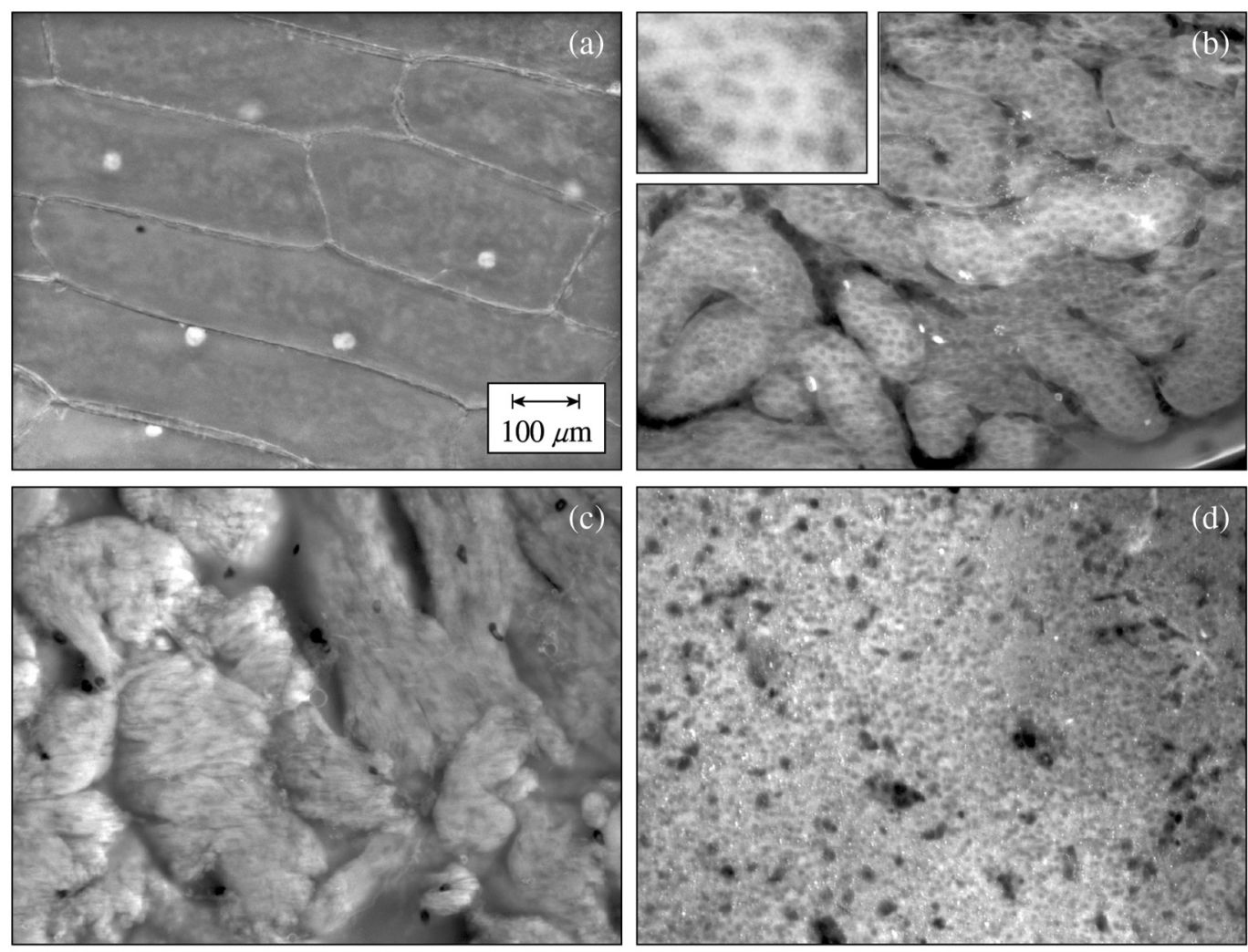

G11849J1

Fig. 2 Images obtained with MUSE autofluorescence: (a) fresh white onion tissue, (b) fresh frog kidney tissue, (c) fresh frog cardiac muscle tissue, and (d) frog liver tissue.
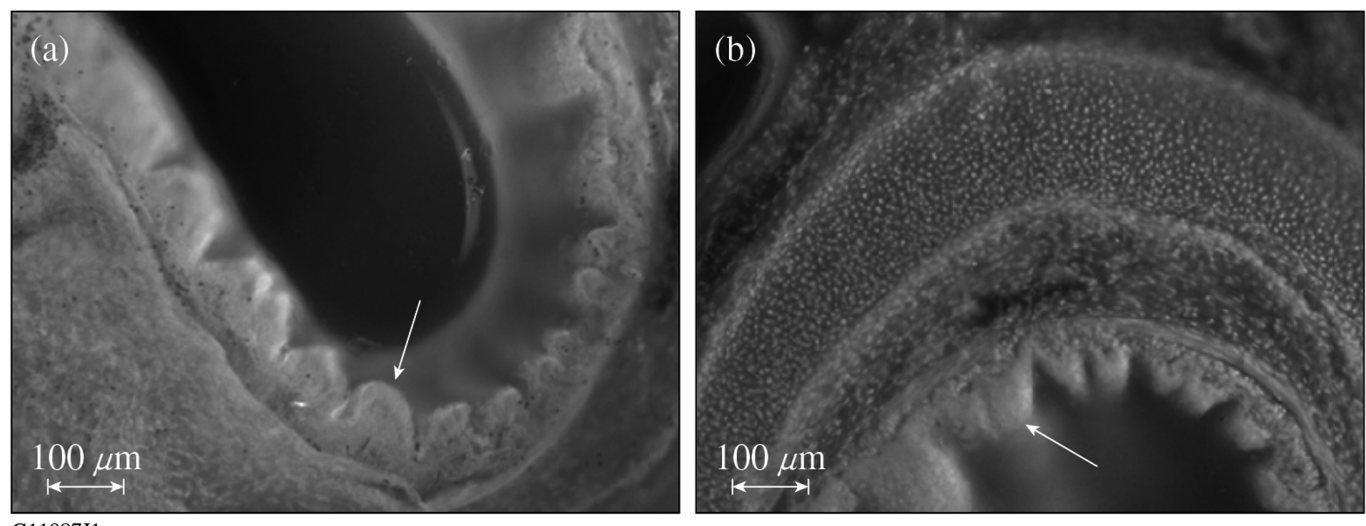

G11887J1

Fig. 3 Preserved porcine bronchi (a) unstained and (b) after a brief immersion in an aqueous solution of Hoechst 33342.

33342 for $15 \mathrm{~s}$ to enhance the prominence of cell nuclei. Although autofluorescence imaging demonstrated the folded epithelial layer (arrow) and underlying smooth muscle, nuclear staining yielded additional details and enabled us to visualize what appears to be a cartilage plate.

The emission spectra of each dye must have distinct spectral peaks if they are to be used in combination to differentiate cellular components in color imaging. Both DAPI (4', 6-Diamidino-2-Phenylindole, Dihydrochloride) and Hoechst 33342 stain the nucleus and emit in the blue spectral region; eosin $\mathrm{Y}$ and fluorescein sodium stain cytoplasm and emit in the yellow and red regions. The concentration of each dye must be optimized to achieve cellular component differentiation after a fixed immersion duration and fixed exposure duration; sequential staining can be performed to determine optimal concentrations for a single immersion staining mixture. A minimum concentration is necessary to achieve staining, but overstaining results in the reduction or complete loss of necessary image contrast (Fig. 4) and/or the introduction of image artifacts. Figure 4(a) is an understained tissue, where the blue autofluorescence signal is roughly equivalent to that of the yellow stain $(500 \mu \mathrm{g} / \mathrm{mL}$ for $60 \mathrm{~s})$. Overstaining as in Fig. 4(b) $(6.25 \mathrm{mg} / \mathrm{mL}$ for $60 \mathrm{~s})$ results in a nonuniform and indistinct appearance of cellular structure with multiple artifacts. 

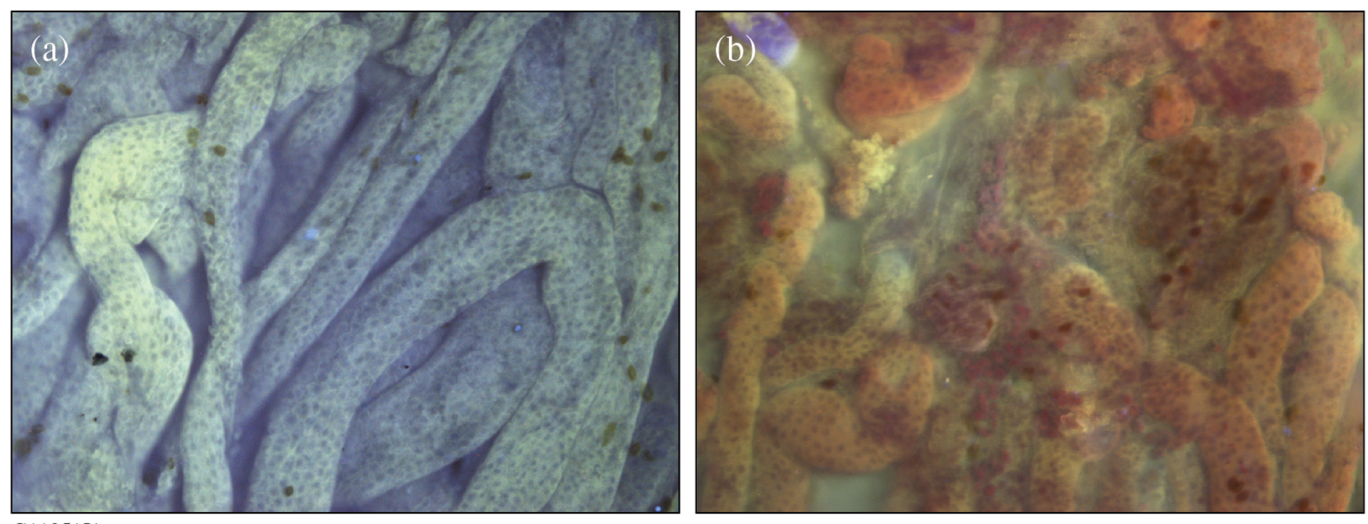

Fig. 4 Eosin $Y(a)$ understained and (b) overstained ranine kidney.

Dyes can be selected to achieve a desired anatomical detail coloration. The kidney tissue samples in Figs. 5(a) and 5(b) used propidium iodide $(500 \mu \mathrm{g} / \mathrm{mL}$ for $15 \mathrm{~s})$ and Hoechst 33342 $(500 \mu \mathrm{g} / \mathrm{mL}$ for $15 \mathrm{~s})$ for nuclear staining red and blue, respectively. Note the barely visible blue autofluorescence of the cytoplasm. Eosin Y exposed the cytoplasm of the cells [Fig. 5(c)]; nuclei are evident as darker features. Better imaging [Fig. 5(d)] was achieved by successively immersing the tissue sample in eosin $\mathrm{Y}(5 \mathrm{mg} / \mathrm{ml}$ in water for $40 \mathrm{~s})$ and Hoechst 33342 $(500 \mu \mathrm{g} / \mathrm{mL}$ in water for $40 \mathrm{~s})$; note simultaneous differential colorization of cytoplasm and nuclei (yellow and blue, respectively). Glomeruli and tubules were readily appreciated after $<5$ min of total sample preparation (including cutting, staining, and imaging).

\subsection{Digital Image Processing}

Image acquisition arising from fluorescence by multiple stain using a color camera, in effect, records the red, green, and blue spectral image components with an eight-bit resolution, i.e., with intensity ranging from 0 to 255 . To optimally generate MUSE images with multiple fluorophores, the fluorescence intensity of each dye should be roughly equivalent within this limited range. Because the necessary duration of UV light exposure is the same for all dyes, the achieved fluorescence from each dye must be optimized by adjusting the staining parameters (concentration and exposure duration); this can be done sequentially or by optimizing a mixture solution for a single immersion. The resultant color image subsequently can be
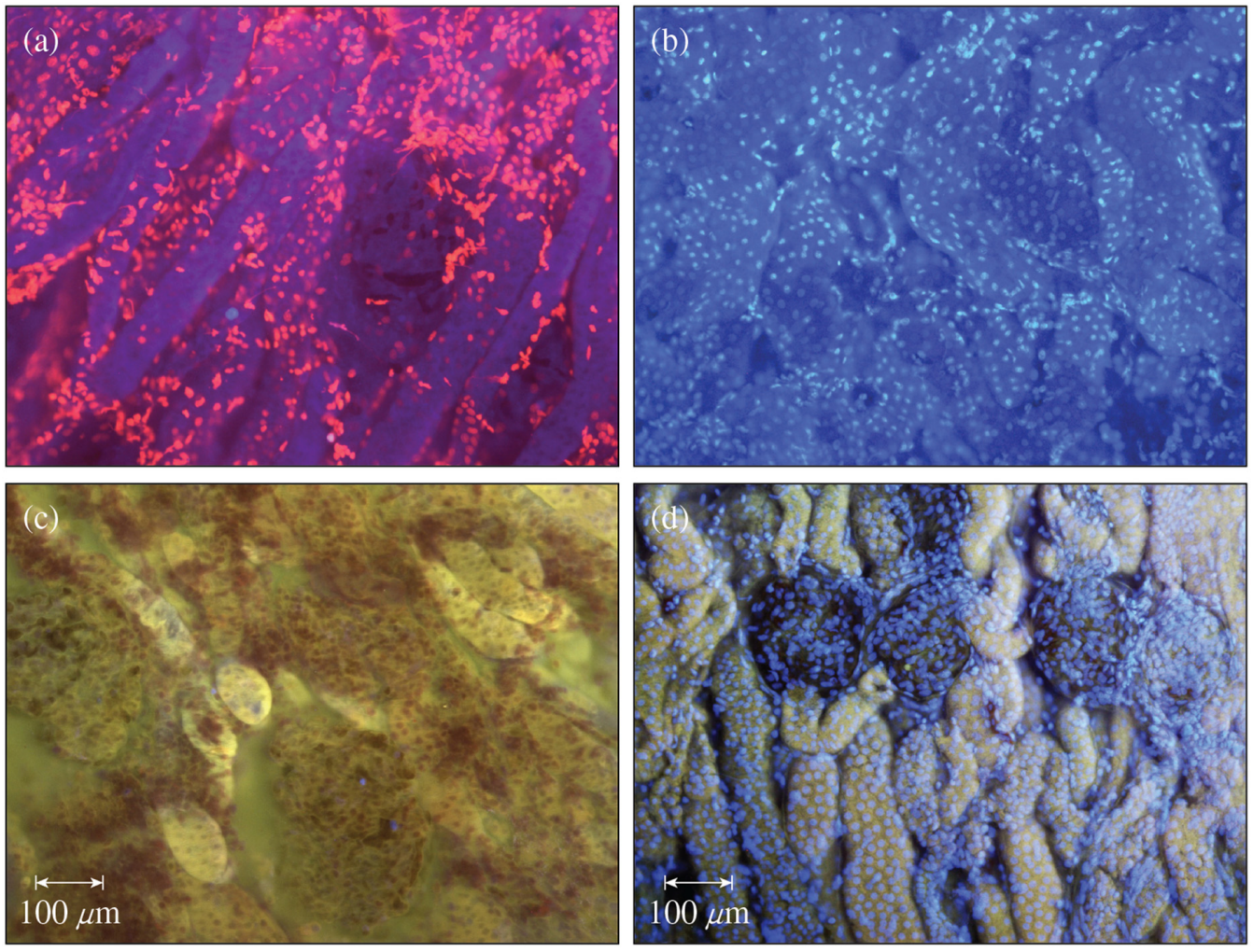

G11850J1

Fig. 5 Ranine kidney stained with (a) propidium iodide, (b) Hoechst 33342, (c) eosin Y, and (d) Hoechst 33342 and eosin $\mathrm{Y}$. 

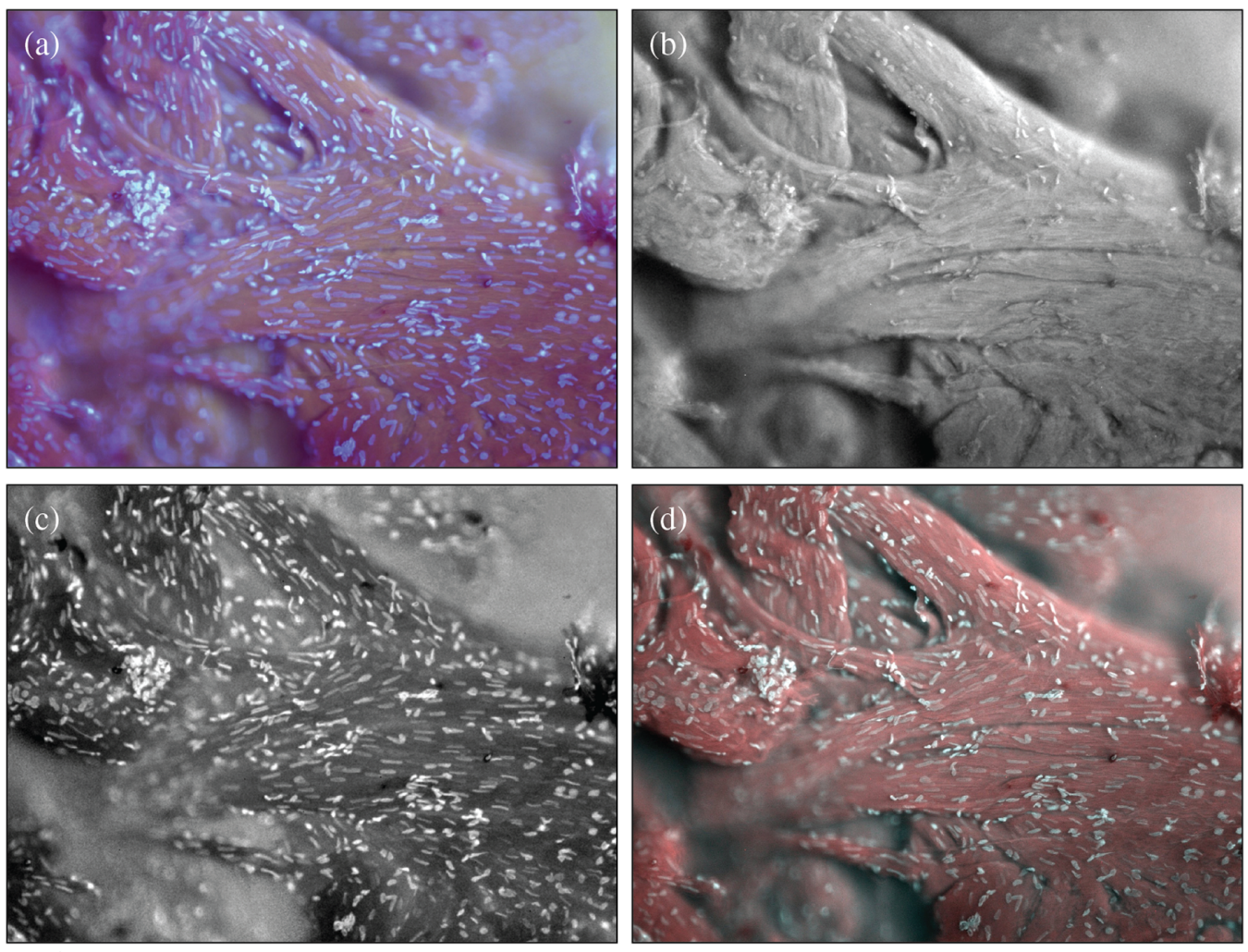

G11852J1

Fig. 6 (a) MUSE fresh ranine cardiac muscle tissue under staining with Hoechst 33342 and eosin Y. Digitally processed images using the original blue, green and red image components of the RGB color image to separate out the emission components of (b) eosin $Y$ and (c) Hoechst 33342.

(d) Color enhanced and digitally processed to improve the image quality.

examined as separate monochrome images and independently manipulated and recombined. Cardiac tissue exposed sequentially to $2 \mathrm{mg} / \mathrm{mL}$ of eosin and $500 \mu \mathrm{g} / \mathrm{mL}$ of Hoechst for $1 \mathrm{~min}$ each result in nuclear fluorescence in blue and green channels and cytoplasmic fluorescence in green and red [Fig. 6(a)]. A number of different methods can then be used to separate the two signal components (such as subtraction or division between the constituent images of the blue, green and red channels). This is demonstrated in the images shown in Figs. 6(b) and 6(c), where the image components of the eosin and Hoechst, respectively, are separated. Furthermore, the images can be recombined to form new images with tailor color designation, such as in the example image shown in Fig. 6(d).

Digital image processing can be used to enhance the image quality and visualization of features. This is demonstrated in the images shown in Fig. 7. Separating the channels of the original ranine liver [Fig. 7(a)] reveals image degradation resulting from blue channel saturation and red channel underexposure. Increasing red channel intensity and replacing the blue channel with the green channel results in a new image [Fig. 7(b)] that provides a clearer visualization of tissue microstructure.

\subsection{Evaluation of Mixed Fluorescent Staining Procedures}

In life-science educational settings, the focus is on understanding cellular and tissue structure and not on the techniques of tissue preparation; consequently, the most rapid and simple tissue preparation method is highly desirable. A premixed powder
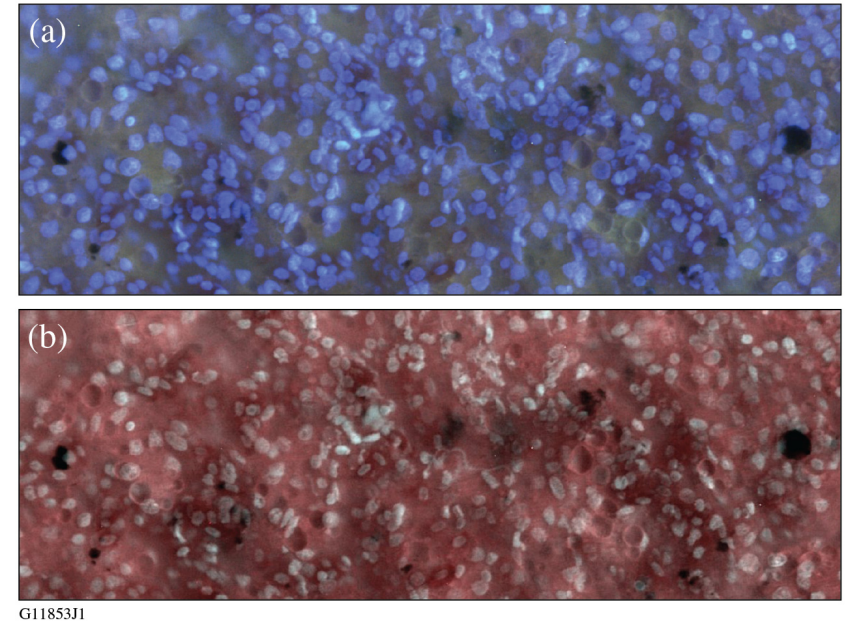

Fig. 7 (a) Hoechst 33342 and eosin $Y$ stained ranine liver and (b) blue and green channels interchanged.

pellet or capsule with appropriate dye concentrations that can be dissolved in a fixed volume of distilled water or saline in 1 to 2 min would be ideal. As this results in a single fixed-duration immersion in the dye solution, the dye concentrations must be optimized.

To explore this concept, we arrived at a mixture of eosin $\mathrm{Y}$ and Hoechst 33342 powders in a 4:1 ratio by weight and placed $250 \mathrm{mg}$ in size 00 gelatin capsules $(0.9 \mathrm{~mL})$. The capsule can be opened but is also readily soluble if one stirs in $100 \mathrm{~mL}$ of 
deionized water; this results in an abundant supply for a classroom session. A 60-s immersion yielded images indistinguishable from separate dye immersions. When using fresh tissue specimens, a brief exposure to isopropanol enhanced the nuclear staining. The entire procedure from harvesting the tissue sample followed by a 10-s phosphate-buffered saline (PBS) rinse, $20 \mathrm{~s}$ in isopropanol, a 10-s PBS rinse, $60 \mathrm{~s}$ in a stain solution, and a 10 -s rinse-to-image capture takes $<3 \mathrm{~min}$. We estimate that five tissues could be studied and discussed by each student in a typical class laboratory.
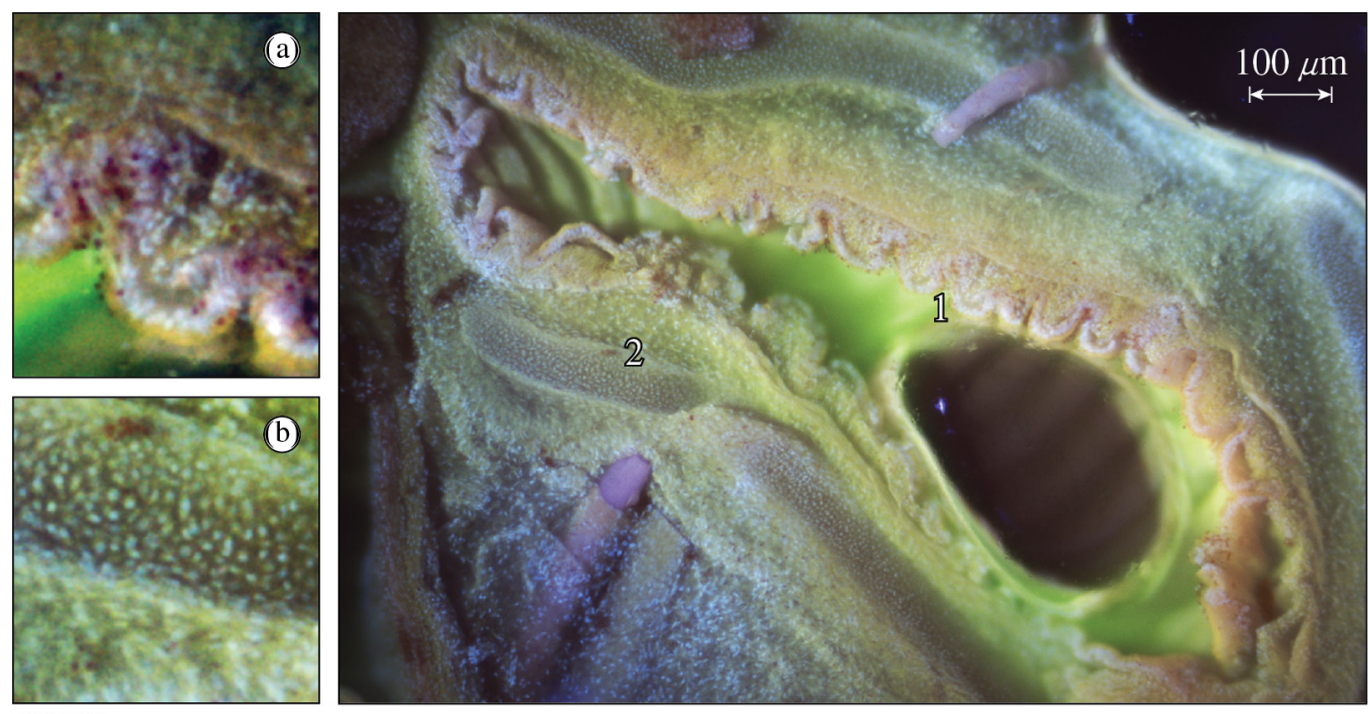

G11854J1

Fig. 8 MUSE images of preserved pig bronchial tissue using a $4 \times$ magnification microscope objective. Insets are $3 \times$ magnified portions of the image at numbered locations showing (a) an area of folded epithelia (lower part) and smooth muscle tissue (upper part) and (b) an area of smooth muscle tissue (lower part) and cartilage (upper part).
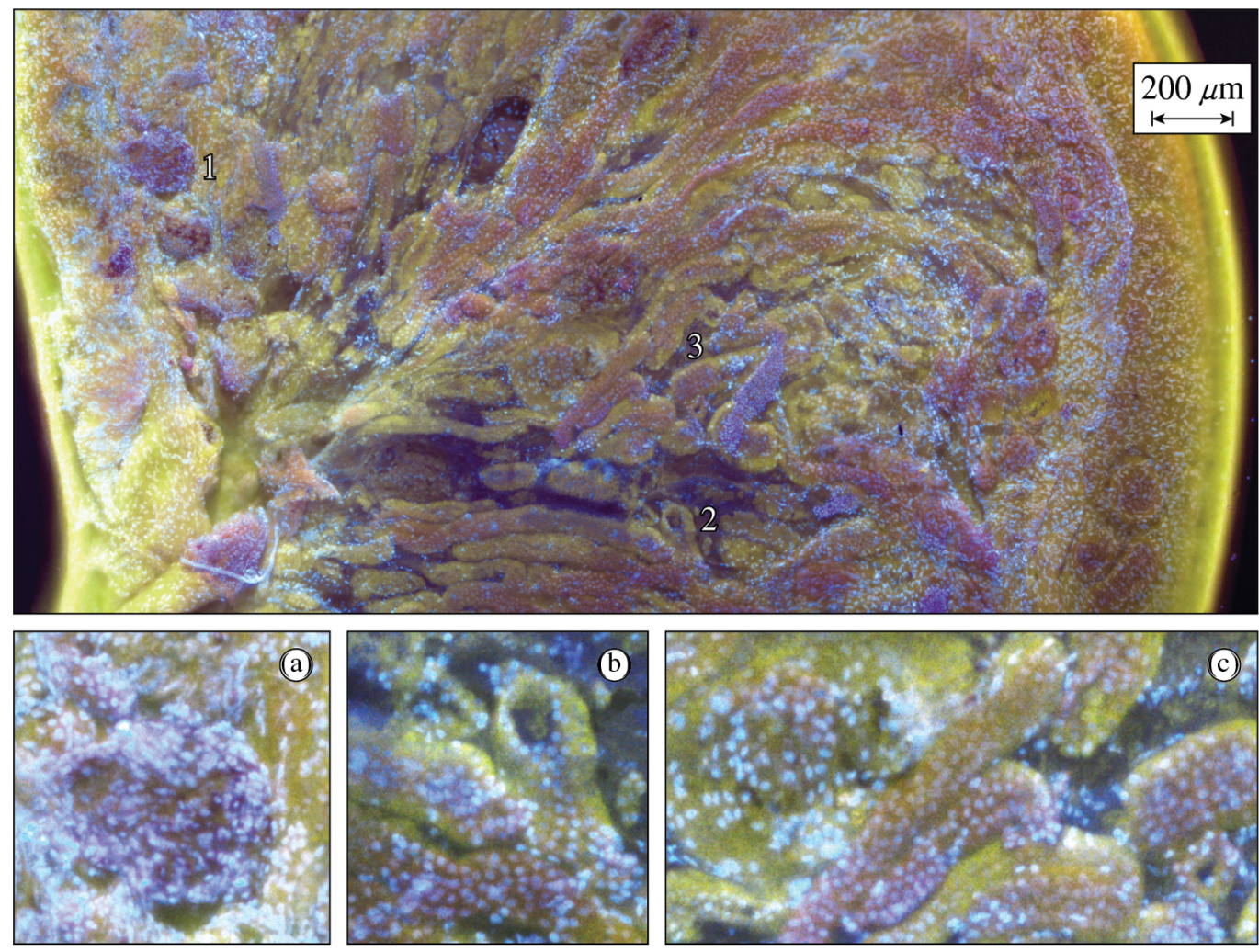

G11855J1

Fig. 9 Stitched wide-field image of ranine kidney. The images shown as insets, denoted 1, 2, and 3, are $3 \times$ magnifications of the stitched image at the noted locations showing (a) a glomerulus and ( $b$ and $c)$ parts of the uriniferous tubule structure. 


\subsection{Imaging Format Considerations}

Unlike clinical pathology settings where high spatial resolution is routinely generated for conclusive diagnostic purposes, in educational settings the goal is to provide initial insight into normal tissue structure. An MUSE image of preserved fetal porcine bronchial tissue provides a dramatic example of tissue structure at lower spatial resolution using a $4 \times$ international standard microscope objective in combination with a color CMOS camera with $2.4-\mu \mathrm{m}$ pixel size (Fig. 8).

Although providing a larger field of view is clearly beneficial, it is also desirable to maintain high-resolution detail. The trade-off between providing a large field of view and maintaining adequate spatial resolution can be negated by employing digital stitching. This is demonstrated in Fig. 9, where a cross section of frog kidney is shown obtained by digitally stitching (using Adobe Photoshop) five individual images acquired with a $4 \times$ microscope objective to create a large image that provides high resolution throughout the image field. The magnified images indicated good visualization of nuclei organization using the $4 \times$ objective.

As mentioned in Sec. 2, various cameras incorporating different CCD and CMOS sensors were tested as part of this work. These cameras are commercially available and are relatively inexpensive, representing current generation products. In general, all cameras performed adequately, providing images that are generally indistinguishable to a nonexpert such as those shown in Figs. 8 and 9. Smaller pixels support lower loss of optical resolution resulting from digital image acquisition. Active camera cooling provides a higher signal-to-noise ratio, which is also highly dependent on the sensor quality including color separation and signal-to-noise ratio.

\section{Discussion}

Creating the required workforce for a knowledge-based economy and fostering individual talent are of fundamental importance to future developments in the life sciences and biotechnology. The creation of the next-generation talent starts from early school years and warrants better curricular development. MUSE imaging provides a novel tool likely to enhance life-science education by providing information-rich images of tissue microstructure with minimal difficulty and perquisite student skills. Sample preparation is safe, efficient, and familiar to students with minimal chemistry or biology lab experience. When desired, nontoxic fluorescent dyes that selectively highlight cellular compartments can be used productively to enhance image contrast and make anatomical detail more readily apparent.

MUSE-based labs can also provide cross-disciplinary experiences in STEM education, i.e., in conjunction with physics, chemistry, and computer science curricula. Students can collect their own images and cross reference them to texts to identify the observed structures. As images are provided in real time, students also have the opportunity to locate structures of specific interest in the classroom or text. This rapid and safe methodology promises to provide rewarding early experiences in the classroom that can help generate and sustain the interest of students, the next generation of our scientists and medical professionals.

Development of suitable curricula would be the focus of future work. Limited experience to date suggests that students can directly associate MUSE images with textbook images such as $H \& E$ slides or schematic representations of the microstructure of tissues and organs. Moreover, as it has been previously discussed, ${ }^{13}$ MUSE images can be color mapped to virtual $H \& E$ appearances using image processing that can be performed in near-real time. Such conversion generates images that can closely approximate the authentic H\&E appearance and can therefore enable direct comparison of MUSE images with textbook images. Additional work to develop a MUSE-based image database can further enhance the choices available to students through well-designed curricula.

It must be noted that some of the concepts related to the translation of MUSE imaging in the education setting might be relevant to the application of MUSE in low-resource settings to address medical applications. For this application, low-cost optics in combination with portable electronic devices (laptop computers or tablets) and low-power LED illumination can provide a new way to address diagnostic issues by providing a lowcost alternative to conventional $\mathrm{H} \& \mathrm{E}$ staining.

\section{Disclosures}

This report was prepared as an account of work sponsored by an agency of the U.S. Government. Neither the U.S. Government nor any agency thereof, nor any of their employees, makes any warranty, express or implied, or assumes any legal liability or responsibility for the accuracy, completeness, or usefulness of any information, apparatus, product, or process disclosed, or represents that its use would not infringe privately owned rights. Reference herein to any specific commercial product, process, or service by trade name, trademark, manufacturer, or otherwise does not necessarily constitute or imply its endorsement, recommendation, or favoring by the U.S. Government or any agency thereof. The views and opinions of authors expressed herein do not necessarily state or reflect those of the U.S. Government or any agency thereof.

\section{Acknowledgments}

We thank K. L. Marshall for his support in selection of dyes that meet classroom safety requirements and recommending appropriate chemical safety and handling procedures. The information, data, or work presented herein was funded in part by the Advanced Research Projects Agency-Energy (ARPA-E), U.S. Department of Energy, under Award Number DEAR0000568, the Department of Energy National Nuclear Security Administration under Award Number DENA0001944, the University of Rochester, and the New York State Research and Development Authority. The support of the DOE does not constitute an endorsement by the DOE of the views expressed in this paper.

\section{References}

1. M. U. Smith and A. C. H. Kindfield, "Teaching cell division: basics \& recommendations," Am. Biol. Teach. 61(5), 366-371 (1999).

2. C. R. Brown, The Effective Teaching of Biology, 1st ed., E. Perrott, Ed., Effective Teacher Series, Longman Group Limited, Harlow, United Kingdom (1995).

3. C. Keller, "Two-year community: using formative assessment to improve microscope skills among urban community college general biology I lab students," J. Coll. Sci. Teach. 46(3), 11-18 (2017).

4. A. Wigfield and J. S. Eccles, "Expectancy-value theory of achievement motivation," Contemp. Educ. Psychol. 25(1), 68-81 (2000).

5. J. Oakley, "Science teachers and the dissection debate: perspectives on animal dissection and alternatives," Int. J. Environ. Sci. Ed. 7(2), 253267 (2012). 
6. R. H. Webb, "Confocal optical microscopy," Rep. Progr. Phys. 59(3), 427-471 (1996)

7. L. Schermelleh, R. Heintzmann, and H. Leonhardt, "A guide to superresolution fluorescence microscopy," J. Cell. Biol. 190(2), 165-175 (2010).

8. P. J. Campagnola and C.-Y. Dong, "Second harmonic generation microscopy: principles and applications to disease diagnosis," Laser Photonics Rev. 5(1), 13-26 (2011).

9. P. T. C. So et al., "Two-photon excitation fluorescence microscopy," Ann. Rev. Biomed. Eng. 2(1), 399-429 (2000).

10. J.-X. Cheng and X. S. Xie, "Coherent anti-stokes Raman scattering microscopy: instrumentation, theory, and applications," J. Phys. Chem. B 108(3), 827-840 (2004).

11. J. M. Schmitt, "Optical coherence tomography (OCT): a review," IEEE J. Sel. Top. Quantum Electron. 5(4), 1205-1215 (1999).
12. D. A. Orringer et al., "Rapid intraoperative histology of unprocessed surgical specimens via fibre-laser-based stimulated Raman scattering microscopy," Nat. Biomed. Eng. 1, 0027 (2017).

13. F. Fereidouni et al., "Microscopy with ultraviolet surface excitation for rapid slide-free histology," Nat. Biomed. Eng. 1(12), 957-966 (2017).

14. B. Lin et al., "Real-time microscopic imaging of esophageal epithelial disease with autofluorescence under ultraviolet excitation," Opt. Express 17(15), 502-509 (2009).

15. R. N. Raman et al., "Evaluation of the contribution of the renal capsule and cortex to kidney autofluorescence intensity under ultraviolet excitation," J. Biomed. Opt. 14(2), 020505 (2009).

Biographies for the authors are not available. 